\title{
Role of Bcl-2/adenovirus E1B 19 kDa-interacting protein 3 in myocardial cells in diabetes
}

\author{
WENZHONG ZHOU* ${ }^{*}$, JIAN YANG ${ }^{*}$, DI ZHANG, FENGYIN LI, GUO LI, YANYUN GU and MIN LUO \\ Department of Endocrine and Metabolic Diseases, Shanghai Institute of Endocrine and Metabolic Diseases, \\ Ruijin Hospital, Jiaotong University School of Medicine, Shanghai 200025, P.R. China
}

Received July 18, 2014; Accepted February 18, 2015

DOI: $10.3892 /$ etm.2015.2439

\begin{abstract}
Cardiovascular complications are the major causes of morbidity and mortality associated with Type 2 Diabetes. Among the macrovascular complications, diabetic cardiomyopathy (DCM) is generally considered to be inadequately recognized and managed. Bcl-2/adenovirus E1B 19 kDa-interacting protein 3 (BNIP3), is known to play a key role in the initiation of the mitochondrial pathway of apoptosis induced by hypoxia and acidosis in the heart. It is unknown whether BNIP3 is also important for cardiac cell survival or adaption in response to hyperglycemia. Based on the previous finding that BNIP3 was significantly induced in the diabetic rat heart, BNIP3 was transfected in primary rat cardiomyocytes and the H9c2 cell line in the present study. Overexpressed BNIP3 decreased the mitochondrial membrane potential and induced cell apoptosis. When BNIP3 was knocked down, the effect on cell apoptosis was reversed. Transcriptome analysis showed that the genes regulating mitochondrial metabolism, such as carnitine palmitoyltransferase $1 b$, cytochrome $c$ oxidase subunit VIIIb and creatine kinase (brain), and those regulating cardiac fibrosis, such as matrix metallopeptidase 9, could be the targets of BNIP3 in rat cardiomyocytes. In conclusion, hyperglycemia-induced BNIP3 expression may compromise cardiac cell survival and function. Under the diabetic condition, BNIP3 could be involved in the regulation of mitochondrial function, lipid metabolism and fibrosis. BNIP3 could therefore serve as a potential drug target against diabetic macrovascular complications and, in particular, DCM.
\end{abstract}

Correspondence to: Dr Yanyun Gu, Department for Endocrine and Metabolic Diseases, Shanghai Institute of Endocrine and Metabolic Diseases, Ruijin Hospital, Jiaotong University School of Medicine, 197 Ruijin Er Road, Shanghai 200025, P.R. China

E-mail: guyanyun@hotmail.com; gyy11251@rjh.com.cn

*Contributed equally

Key words: diabetes, diabetic cardiomyopathy, Bcl-2/adenovirus E1B $19 \mathrm{kDa}$-interacting protein 3

\section{Introduction}

An estimated 371 million individuals are afflicted with diabetes worldwide, and the incidence is increasing rapidly, particularly in developing countries such as China $(1,2)$. Diabetes doubles the risk of cardiovascular disease (CVD) (3), and CVDs are the leading cause of mortality in individuals with diabetes. Among the most common diabetic macrovascular diseases are myocardial infarction, coronary artery disease and congestive heart failure-diabetic cardiomyopathy (DCM). Glucose control alone does not benefit macrovascular diseases (4) as much as diabetic microvascular complications, suggesting that the pathophysiological mechanism underlying diabetic macrovascular complications remains to be elucidated.

DCM is an occult cardiovascular complication associated with chronic diabetes and is easily overlooked in clinical practice $(5,6)$. DCM has been known as an independent cause of congestive heart failure in the absence of coronary artery disease and hypertension (7). To date, the potential etiologies have included microangiopathy, autonomic neuropathy or disrupted cellular metabolism (8-14). The underlying pathophysiological mechanism of DCM has been associated with blocked intracellular insulin signaling (insulin resistance in cardiomyocytes), metabolic substrate shift, enhanced production of reactive oxygen species, cellular apoptosis and fibrosis in cardiac muscle (8-14); however, in the absence of adequate therapeutic options other than blood glucose control, specific molecular targets or signaling pathways require further exploration and definition.

Bcl-2/adenovirus E1B $19 \mathrm{kDa}$-interacting protein 3 (BNIP3), a pro-apoptotic protein with a Bcl-2 homology 3 (BH3)-only domain, has been suggested to mediate hypoxia or ischemia in cardiac muscle $(15,16)$. The pro-apoptotic $\mathrm{BH} 3$-only proteins play important roles in the pathogenesis of heart failure, cancer and inflammatory diseases $(17,18)$. Compared with other $\mathrm{BH} 3$-only proteins, the effect of BNIP3 does not require the formation of heterodimers with other BH1-4 multidomain B-cell lymphoma 2 proteins. Instead, BNIP3 homodimers insert onto the mitochondrial outer membrane by their transmembrane domains. The increased insertion of BNIP3 homodimers induces mitochondrial permeablization and impaired mitochondrial membrane potential, resulting in damaged mitochondrial function and cellular apoptosis $(19,20)$. Previous studies $(15,21,22)$ have 
suggested that BNIP3 is one of the major proteins mediating ischemia/reperfusion injury of cardiomyocytes, as well as cardiac remodeling. The ablation of BNIP3 has been shown to significantly improve post-infarction cardiomyocyte remodeling and cellular apoptosis (23). Despite this, it is yet to be elucidated whether hyperglycemia-induced cellular apoptosis in DCM is also mediated by BNIP3, and if BNIP3 exerts other effects on cardiac myocytes in response to hyperglycemia.

In our previous studies, BNIP3 expression was shown to increase in the cardiac muscle of a high-fat diet diabetic rat model $(24,25)$, suggesting its role in diabetic cardiovascular complications. These studies have prompted the present study, which aims to further investigate the role of BNIP3 in the diabetic heart and its underlying molecular mechanism.

\section{Materials and methods}

Animal study and maintenance. Animals were purchased from the Shanghai Laboratory Animal Center (Shanghai, China). The animal protocol was reviewed and approved by the Animal Care Committee of Shanghai Jiao Tong University School of Medicine (Shanghai, China). Eight-week-old Sprague Dawley rats were treated with low-dose streptozotocin (STZ) by intraperitoneal injection following a high-fat diet for two months, as described in our previous studies $(24,25)$.

Plasmid construction and transfection. Whole-length BNIP3 was amplified from rat heart cDNA using the following primers: Forward, 5'-CTCGAGTTTGCGGAGCCACC-3'; and reverse, 5'-GGATCCTCAGAAGGCAGATCCAAG-3'. The amplified product was then cloned into a pIRES-EGFP vector (BD Biosciences, San Jose, CA, USA) and confirmed by restriction enzyme analysis and DNA sequencing. Effectene ${ }^{\circledR}$ (Qiagen, Hilden, Germany) was used as a transfection reagent in the primary cardiac cell culture, while SuperFect (Qiagen) was used as a transfection reagent both for plasmid and small interfering RNA (siRNA) in H9c2 cells (American Type Tissue Collection, Manassas, VA, USA).

siRNA. The sequence of the BNIP3 siRNA was as follows: Forward, 5'-TCAGCATGAGAAACACAAGCGT-3'; and reverse, 5'-TCCCCAATCCAATGGCTAACAG-3'. The sequence of the control siRNA was as follows: Forward, 5'-UUCUCCGAACGUGUCACGUTT-3'; and reverse, 5'-ACGUGACACGUUCGGAGAATT-3'. siRNA was synthesized by GenePharma Co., Ltd. (Shanghai, China).

Primary cardiac cell culture. Hearts of newborn rats (one-to-two days old) were dissected and washed in icy D-Hank's solution $\left(0.4 \mathrm{~g} / 1 \mathrm{KCl} ; 60 \mathrm{mg} / 1 \mathrm{KH}_{2} \mathrm{PO}_{4} ; 0.35 \mathrm{~g} / 1 \mathrm{NaHCO}_{3}\right.$; $80 \mathrm{~g} / \mathrm{ml} \mathrm{NaCl} ; 48 \mathrm{mg} / 1 \mathrm{Na}_{2} \mathrm{HPO}_{4}$. The isolated hearts were minced and digested with $0.125 \%$ trypsin mixed with $0.01 \%$ EDTA, and Type II collagenase (Thermo Fisher Scientific, Inc., Waltham, MA, USA) in a $37^{\circ} \mathrm{C}$ water bath with minor agitation at $50 \mathrm{rpm}$. Digestion was terminated by $20 \%$ calf serum-Dulbecco's Modified Eagle's Medium (DMEM; Thermo Fisher Scientific, Inc.) with $5.6 \mathrm{mM}$ glucose. The digested cells were washed and then preincubated in $5 \% \mathrm{CO}_{2}$ at $37^{\circ} \mathrm{C}$ for $60-90 \mathrm{~min}$ to remove the fibroblasts. Following preincubation the supernatant was transferred with the suspended cells into tissue culture with $0.1 \mathrm{mM}$ bromodeoxyuridine (Sigma-Aldrich, St. Louis, MO, USA). After $12 \mathrm{~h}$, the media were changed with $1 \%$ bovine serum albumin-DMEM (Gibco-BRL, Carlsbad, CA, USA)/M199 (Sigma-Aldrich) mixed media at a 4:1 ratio, along with $500 \mathrm{ng} / \mathrm{ml}$ transferrin, non-essential amino acids (Thermo Fisher Scientific, Inc.) and $25 \mathrm{mM}$ glucose for $48 \mathrm{~h}$.

Cell apoptosis. Annexin V labeling was performed based on the manufacturer's instructions (BD Biosciences). The cells were serum-starved under different glucose concentrations overnight prior to labeling. Annexin V staining was then detected and analyzed using flow cytometry (FACSCalibur ${ }^{\mathrm{TM}}$; BD Biosciences).

Microarray and quantitative polymerase chain reaction $(q P C R)$ analysis. RNA was extracted by using of TRIzol ${ }^{\circledR}$ (Life Technologies) from the cultured cells. cRNA was synthesized and blotted with an Affymetrix rat GeneChip ${ }^{\circledR} 2.0$ array. Reverse transcription was performed using Superscript III Reverse Transcriptase (Life Technologies), according to manufacturer's instructions. Data were then confirmed using qPCR analysis with SYBR ${ }^{\circledR}$ Green (Takara Bio, Inc., Shiga, Japan) on an ABI 7300 Real-Time PCR system (Applied Biosystems, Foster City, CA, USA). The data were analyzed for target gene expression using the $-\Delta \Delta \mathrm{CT}$ method.

Mitochondrial staining and fractional extraction. The mitochondria were stained using MitoTracker ${ }^{\circledR}$ Red CM (ROX) (Thermo Fisher Scientific, Inc.), according to the manufacturer's instructions with minor modifications. Images were captured using an LSM510 confocal microscope (Carl Zeiss AG, Oberkochen, Germany). Mitochondrial isolation was performed in accordance with the instructions provided by Biovision, Inc. (Mountain View, CA, USA). Briefly, cells were homogenized using Dounce tissue grinders (Sigma-Aldrich) to isolate intact mitochondria, from which the mitochondrial protein was extracted.

Western blot analysis. Cellular or mitochondrial protein was loaded on SDS-PAGE gels, separated electrophoretically and transferred to a nitrocellulose membrane with an MP3 device from Bio-Rad Laboratories, Inc. (Hercules, CA, USA). Anti-rabbit BNIP3 antibody was synthesized in our laboratory at a dilution of 1:8,000. In brief, the whole-length BNIP3 cDNA was cloned into the pGEX-4T2 plasmid (Promega Corp., Madison, WI, USA). The pGEX-4T2-BNIP3 plasmid was subsequently transformed into the $E$. coli BL21 strain and induced by $1.0 \mathrm{mmol} / 1$ isopropyl- $\beta$-D-thiogalactopyran oside, with agitation, at $18^{\circ} \mathrm{C}$ overnight. Following transformation, glutathione S-transferase (GST) fusion protein was purified using a B-PER GST Purification kit (Thermo Fisher Scientific, Inc.). Following confirmation using Tandem mass spectrometry (Thermo Fisher Scientific, Inc.), the purified protein was used to immunize New Zealand white rabbits (Shanghai Laboratory Animal Center, Shanghai, China) without the GST tag.

Statistical analysis. All comparisons were analyzed with SPSS 11.0 software (SPSS Inc., Chicago. IL, USA). An independent sample t-test was used to calculate the statistical 

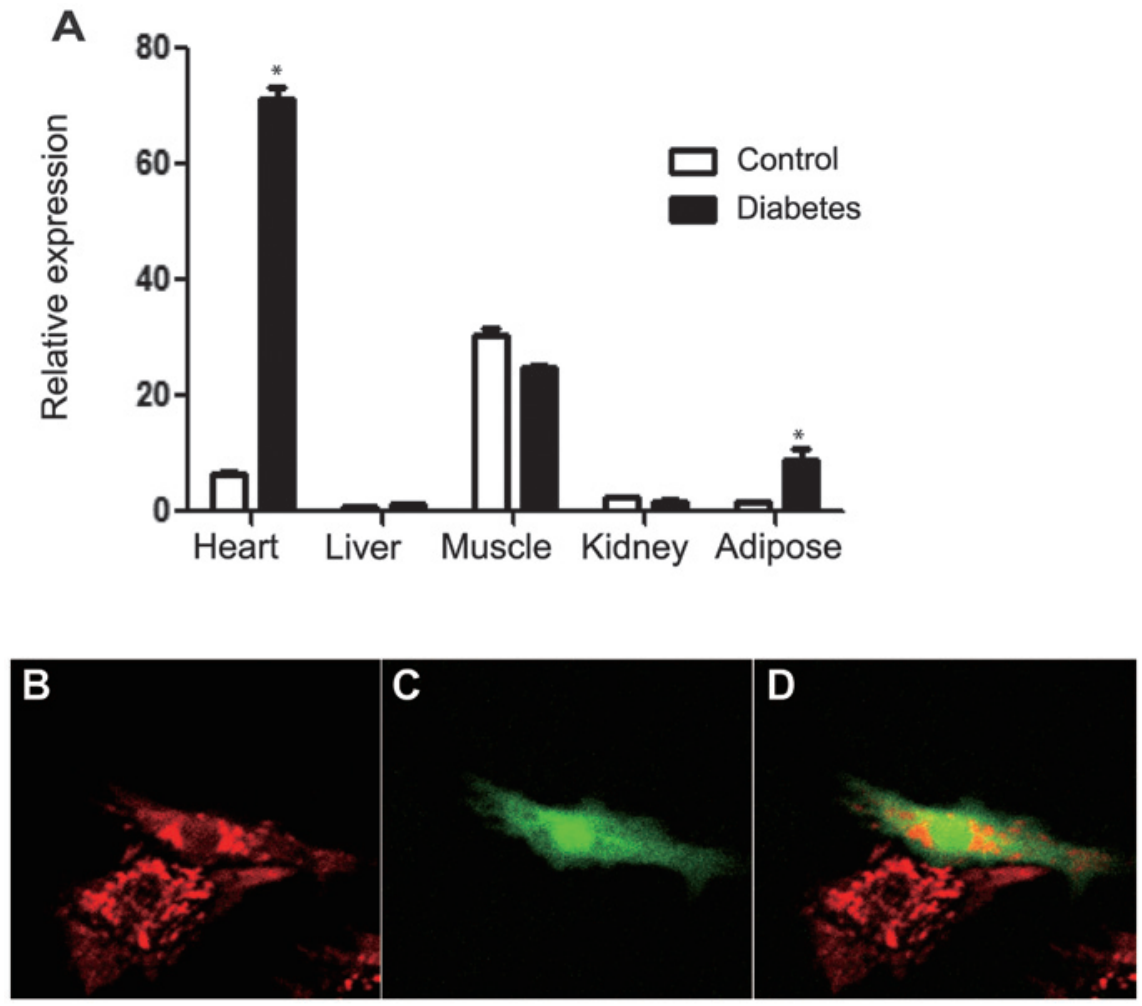

Figure 1. BNIP3 is enriched in heart and skeletal muscles, and BNIP3 overexpression with GFP fluorescence in cultured primary cardiomyocytes leads to mitochondrial fusion. (A) Relative expression of BNIP3 by quantitative polymerase chain reaction analysis in mouse tissues was normalized using actin expression. In diabetic rats, BNIP3 expression in the heart was increased to levels 10 -fold higher than those in control rats. (B-D) Live staining of mitochondria and GFP fluorescence manifesting BNIP3 overexpression in primary rat heart cell culture: (B) Red indicates MitoTracker ${ }^{\circledR}$ Red live staining to visualize the mitochondria; (C) green indicates GFP live fluorescence, representing BNIP3 overexpression in cells; (D) merged figure of B and C (magnification, 20x10). More mitochondrial fusion was found in the cells positive for BNIP3 overexpression compared with the control cells transfected with GFP. BNIP3, Bcl-2/adenovirus E1B $19 \mathrm{kDa}$-interacting protein 3; GFP, green fluorescent protein.

A

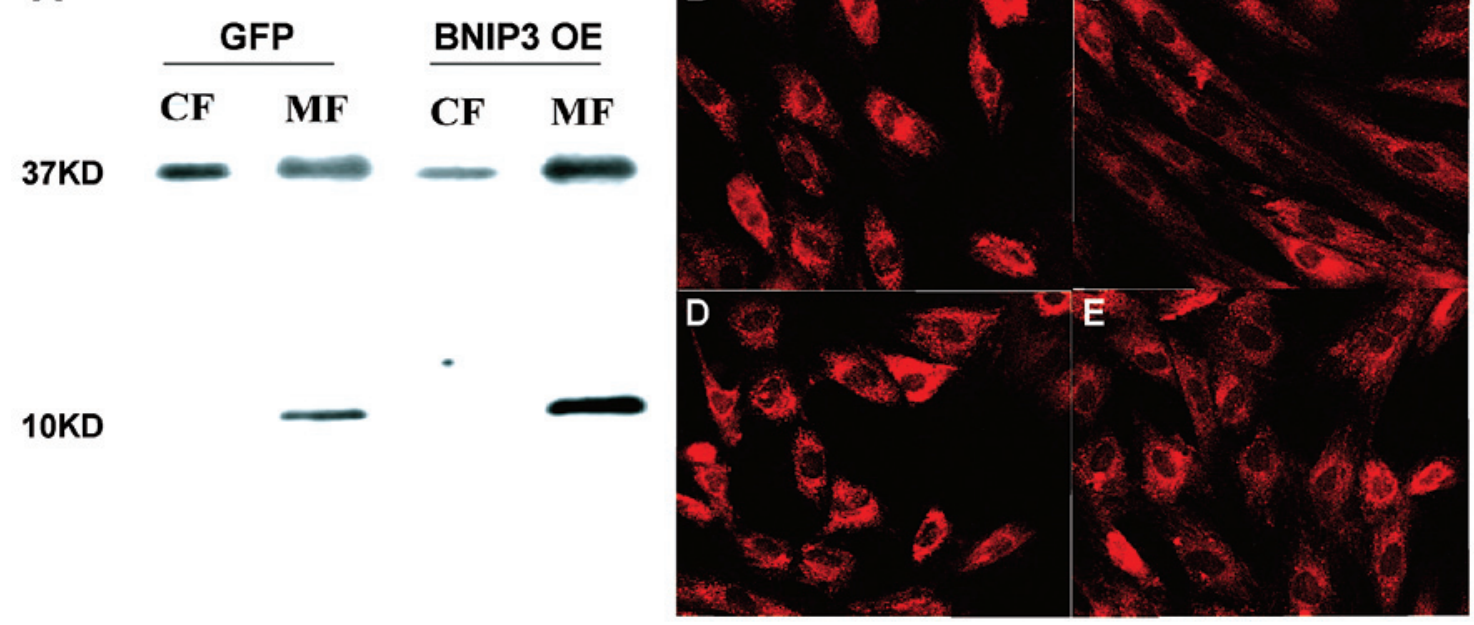

Figure 2. Overexpressed BNIP3 mainly localizes in the mitochondria and leads to diminished mitochondrial membrane potential. (A) Western blotting comparing the BNIP3 protein level in the CF and MF of primary cardiac muscle cell lysates with overexpression of either BNIP3 or GFP. Overexpressed BNIP3 protein was mostly located in the MF. (B-E) Cellular mitochondria staining with MitoTracker ${ }^{\circledR}$ in H9C2 cells with or without BNIP3 overexpression: (B and D) H9C2 cells transfected with GFP plasmid; (C and E) H9C2 cells transfected with BNIP3 plasmid (magnification, 20x10). BNIP3, Bcl-2/adenovirus E1B 19 kDa-interacting protein 3; GFP, green fluorescent protein; CF, cytosolic fraction; MF, mitochondrial fraction; BNIP3 OE, cells transfected with overexpressed BNIP3 plasmid.

significance between two groups and one way analysis of variance was used to make comparisons between multiple groups. $\mathrm{P}<0.05$ was considered to indicate a statistically significant difference.

\section{Results}

BNIP3 is highly expressed in the heart and is significantly induced in diabetes. The expression pattern of BNIP3 in different 


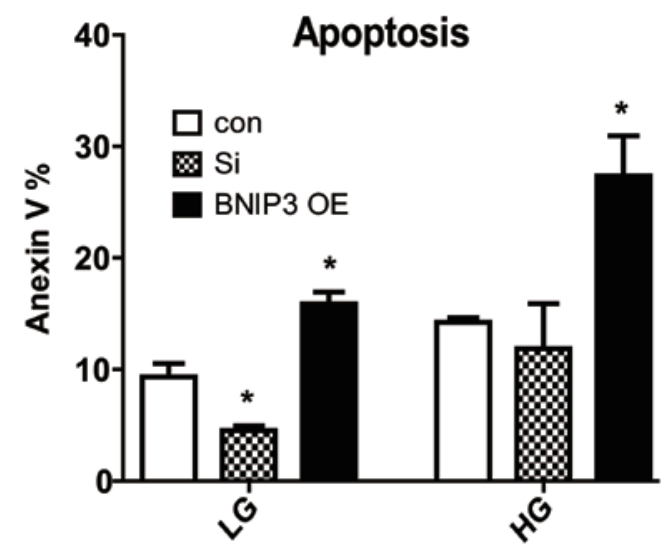

Figure 3. Overexpression of BNIP3 in H9C2 cells significantly induces cellular apoptosis under low- and high-glucose conditions, and inhibition of BNIP3 significantly decreases cellular apoptosis only in the low-glucose condition. Cellular apoptosis was assessed by analyzing Annexin V staining with fluorescence-activated cell sorting $(\mathrm{n}=3)$. " $\mathrm{P}<0.05$ versus the control cells. LG, $5.6 \mathrm{mM}$ glucose concentration; HG, $25 \mathrm{mM}$ glucose concentration; Con, cells transfected with empty plasmid or non-sense siRNA; Si, cells transfected with BNIP3 siRNA; BNIP3 OE, cells transfected with overexpressed BNIP3 plasmid; BNIP3, Bcl-2/adenovirus E1B 19 kDa-interacting protein 3; siRNA, small interfering RNA.
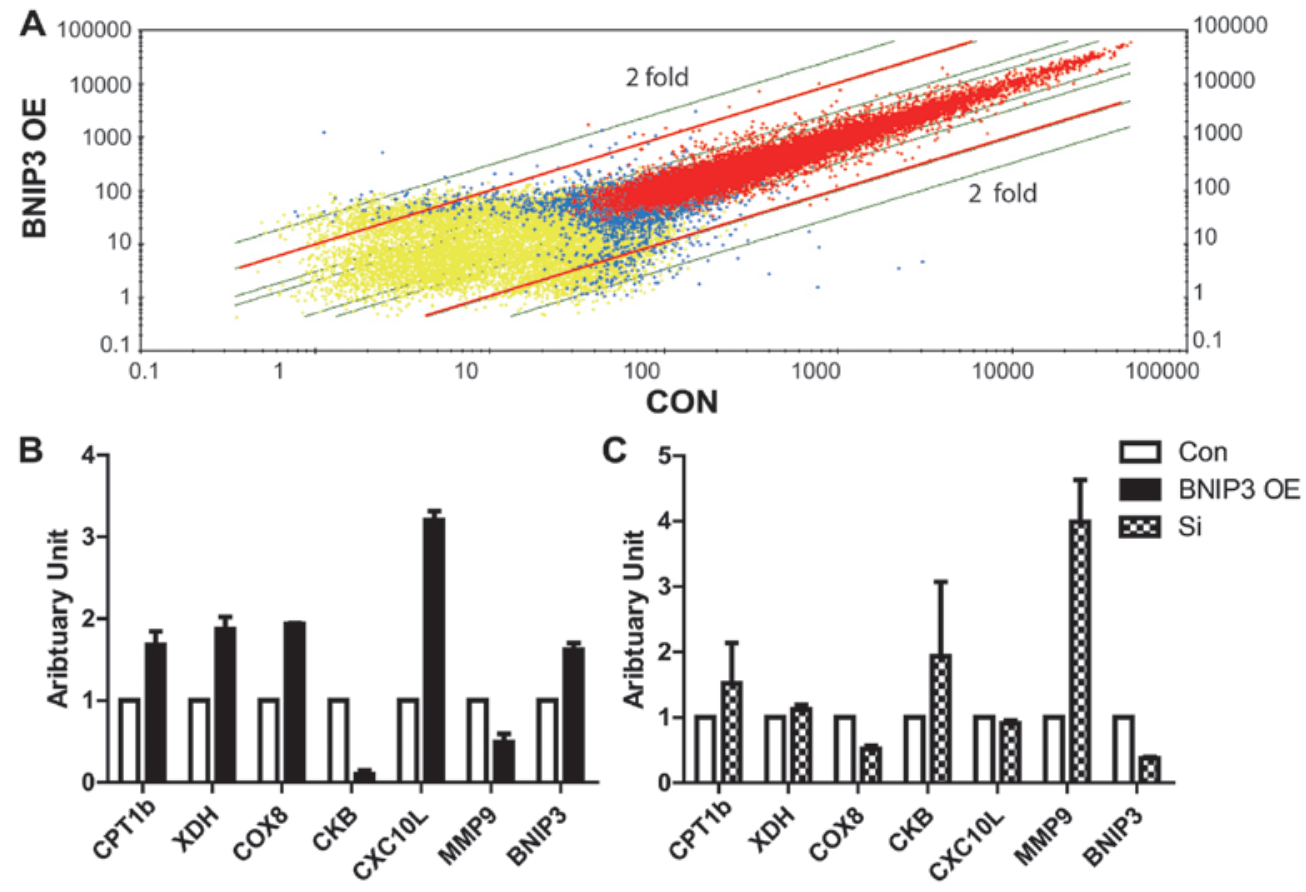

Figure 4. Differential transcription in cardiac cells with BNIP3 overexpression. (A) Microarray analysis of H9C2 cells with overexpression of BNIP3 (BNIP3 OE) or GFP (CON). Genes of interest were selected based on two-fold differences in expression between the BNIP3 OE and GFP transfected samples. (B) Confirmation of relative mRNA expression of BNIP3 downstream targets selected from microarray data with quantitative polymerase chain reaction data $(\mathrm{n}=3)$. (C) Relative expression of BNIP3 downstream target genes in cells transfected with BNIP3 siRNA ( $\mathrm{n}=3)$. Con, cells transfected with empty plasmid or non-sense siRNA; Si, BNIP3 siRNA; BNIP3 OE, cells transfected with overexpressed BNIP3 plasmid; BNIP3, Bcl-2/adenovirus E1B 19 kDa-interacting protein 3; GFP, green fluorescent protein; siRNA, small interfering RNA; CPT1b, carnitine palmitoyltransferase 1b; XDH, xanthine dehydrogenase; COX8, cytochrome $c$ oxidase subunit VIII; CKB, creatine kinase (brain); CXC10L, chemokine (C-X-C motif) ligand 10; MMP9, matrix metallopeptidase 9.

organs was determined using qPCR at the transcriptional level in insulin-sensitive tissues, such as the heart, kidney, liver, muscle and fat. BNIP3 expression was found to be enhanced in the heart, muscle and adipose tissue compared with that in the liver and kidney. In the diabetic rat heart, the BNIP3 mRNA level was further increased to a level 10 -fold higher than that in the non-diabetic control (Fig. 1A). The establishment of the diabetic rat model is described in our previous studies $(24,25)$. These results suggest that BNIP3 is essential for the maintenance of cardiac function in normal and diabetic conditions.
BNIP3 overexpression induces a loss of mitochondrial membrane potential. To investigate the effect of BNIP3 on cardiac muscle, primary cardiac cells were isolated from neonatal rats and transfected with BNIP3-green fluorescent protein (GFP) plasmid one day after ex vivo culturing. Transfected positive cells were shown using GFP fluorescence. Mitochondria were stained red with MitoTracker Red CM (ROX). Confocal imaging showed that mitochondrial fusion was increased in BNIP3-GFP positive cells (Fig. 1B-D). Using the BNIP3 antibody produced 
Table I. Pathway mining to find downstream targets of BNIP3 from mRNA microarray.

\begin{tabular}{|c|c|c|}
\hline Pathway & Gene & Difference compared with CON (fold) \\
\hline \multirow[t]{5}{*}{ Lipid metabolism } & Slc2a4 (Glut4) & $\uparrow 2.30$ \\
\hline & Acsl1 & $\uparrow 2.20$ \\
\hline & Acsl3 & $\downarrow 2.00$ \\
\hline & $\mathrm{Cd} 36$ & $\uparrow 3.00$ \\
\hline & Cpt1b & $\uparrow 2.00$ \\
\hline \multirow[t]{4}{*}{ Mitochondrial oxidative phosphorylation } & Cox $8 b$ & $\uparrow 2.50$ \\
\hline & Capn6 & $\uparrow 2.50$ \\
\hline & $\mathrm{Xdh}$ & $\uparrow 2.50$ \\
\hline & CKB & $\downarrow 2.30$ \\
\hline \multirow[t]{4}{*}{ Cardiac fibrosis } & MMP9 & $\downarrow 3.03$ \\
\hline & MMP12 & $\downarrow 22.67$ \\
\hline & Bdkrb2 & $\downarrow 4.00$ \\
\hline & Klk6 & $\downarrow 3.20$ \\
\hline \multirow[t]{4}{*}{ Calcium metabolism } & $\mathrm{P} 2 \mathrm{rx} 1$ & $\downarrow 8.00$ \\
\hline & Camk2b & $\downarrow 2.82$ \\
\hline & Plcd4 & $\downarrow 2.64$ \\
\hline & Cacna1g & $\uparrow 2.50$ \\
\hline \multirow[t]{4}{*}{ Wnt pathway } & Sfrp1 & $\downarrow 3.48$ \\
\hline & Wif1 & $\downarrow 3.25$ \\
\hline & Axin2 & $\downarrow 3.48$ \\
\hline & Ppp2r2b & $\downarrow 3.73$ \\
\hline \multirow[t]{5}{*}{ Apoptosis and inflammation factors } & Casp12 & $\uparrow 2.14$ \\
\hline & Illa & $\downarrow 3.03$ \\
\hline & VCAM1 & $\downarrow 2.20$ \\
\hline & Pla2g5 & $\uparrow 2.00$ \\
\hline & CXCL10 & $\uparrow 4.60$ \\
\hline \multirow[t]{4}{*}{ Cell cycle } & Cdkn2d & $\downarrow 6.96$ \\
\hline & Ccnb2 & $\downarrow 2.50$ \\
\hline & Pttg1 & $\downarrow 2.00$ \\
\hline & Espl1_predicted & $\downarrow 2.00$ \\
\hline
\end{tabular}

BNIP3, Bcl-2/adenovirus E1B 19 kDa-interacting protein 3; Slc2a4, glucose transporter type 4; Acsl, acyl-CoA synthetase long-chain family member; CD 36, cluster of differentiation 36; Cpt1b, carnitine palmitoyltransferase 1b; Cox8b, cytochrome $c$ oxidase subunit VIIIb; Xdh, xanthine dehydrogenase; Capn6, calpain 6; CKB, creatine kinase (brain); MMP, matrix metallopeptidase; Bdkrb2, bradykinin receptor, $\beta 2$; Klk6, kallikrein 6; P2rx1, purinergic receptor P2X, ligand-gated ion channel, 1; Camk2b, calcium/calmodulin-dependent protein kinase II $\beta$; Plcd4, phospholipase C, $\delta 4$; Cacna1g, calcium channel, voltage-dependent, T type, $\alpha 1 \mathrm{G}$ subunit; Sfrp1, secreted frizzled-related protein 1; Wif1, WNT inhibitory factor 1; Ppp2r2b, protein phosphatase 2, regulatory subunit B (PR 52), $\beta$ isoform; Casp12: caspase 12; Il1a, interleukin 1, $\alpha$; VCAM1, vascular cell adhesion molecule 1; Pla2g5, phospholipase A2, group V; CXCL10, chemokine (C-X-C motif) ligand 10; Cdkn2d, cyclin-dependent kinase inhibitor 2D (p19, inhibits CDK4); Ccnb2, cyclin B2; Pttg1, pituitary tumor-transforming 1; Espl1_predicted, extra spindle poles like 1 (S. cerevisiae) (predicted); CON, H9C2 cells with overexpression of green fluorescent protein.

in our laboratory, it was found that the BNIP3 protein was predominantly localized in the mitochondria instead of the cytosol (Fig. 2). In the H9c2 cell line, MitoTracker Red CM (ROX) staining showed lower mitochondrial membrane potential in the cells that overexpressed BNIP3 compared with the GFP-transfected cells.

Overexpression of BNIP3 causes cellular apoptosis and knockdown of BNIP3 fails to significantly reduce apoptosis in the high-glucose condition. Following the transfection of the H9c2 cells with BNIP3 plasmids for $72 \mathrm{~h}$, it was found that cellular apoptosis was significantly induced in the low(5.6 mM) and high- (25 mM) glucose conditions. When BNIP3 was knocked down, the number of Annexin V-positive cells was significantly reduced in the low-, but not in the high-, glucose culture condition compared with the control (Fig. 3). BNIP3 is therefore sufficient but not necessary for apoptosis in the high-glucose condition.

Comparative transcriptome analysis reveals new downstream signals of BNIP3 targeting oxidative phosphorylation, inflammation and heart remodeling. To further explore the 
downstream targets and effects of BNIP3, along with its role in the apoptosis of heart muscle, mRNA microarray analysis was performed to compare the differential gene expression of GFP and BNIP3-GFP-transfected ex vivo rat heart cells (Fig. 4A). Notably, several genes differentially expressed in the two groups were associated with lipid metabolism, oxidative phosphorylation, inflammation, fibrosis and apoptosis (Table I). qPCR was employed to confirm the data from the microarray analysis. The expression of carnitine palmitoyltransferase $1 \mathrm{~b}$ (CPT1b), cytochrome $c$ oxidase subunit VIIIb (COX8b), xanthine dehydrogenase $(\mathrm{XDH})$ and chemokine $(\mathrm{C}-\mathrm{X}-\mathrm{C}$ motif) ligand 10 was significantly induced by overexpressed BNIP3, while creatine kinase (brain) (CKB) and matrix metallopeptidase 9 (MMP9) expression was inhibited. To further investigate the regulation of these genes by BNIP3, BNIP3 was knocked down by siRNA in the H9c2 cell line. The genes induced by BNIP3 were predominantly reduced following treatment with BNIP3 siRNA, with the exception of CPT1b (Fig. 4B). The genes reduced by the overexpression of BNIP3 were all significantly recovered with BNIP3-knockdown.

\section{Discussion}

In this study, it was found that BNIP3 mRNA expression was induced to a level 10-fold higher in diabetes compared with that in the normal condition. Overexpression and downregulation of BNIP3 led to changes in the cellular apoptosis. Microarray data confirmed that, despite the increased expression of pro-apoptotic genes in diabetes, BNIP3 mediated the effect of chronic hyperglycemia in cardiac cells via multiple pathways.

Comparable with other studies $(26,27)$, overexpressed BNIP3 in the present study reduced the mitochondrial membrane potential, and most overexpressed BNIP3 protein preferentially bound to the outer membrane. The insertion of BNIP3 homodimers opened the mitochondrial permeability transition pore and decreased the mitochondrial membrane potential $(18,28,29)$. Decreased mitochondrial membrane potential indicates mitochondrial dysfunction and apoptosis. In the present study, overexpression of BNIP3 was also shown to induce cellular apoptosis in both low- and high-glucose conditions. The downregulation of BNIP3 using siRNA interference failed to rescue the apoptosis induced by the high glucose levels, suggesting that other signaling mechanisms exist in addition to that involving BNIP3, and that the promotion of apoptosis may not be a major function of BNIP3 in this scenario.

Microarray analysis suggested that BNIP3 targeted genes involved in lipid metabolism and mitochondrial oxidative phosphorylation, such as CPT-1b, COX8b and XDH. CPT1b is a key enzyme mediating lipid transport into the mitochondria for fatty acid oxidation (FAO). COX8H and XDH (30) are crucial regulators of oxidative stress, which is induced by increased FAO and reduced mitochondrial efficiency. CKB, inhibited by BNIP3, is a key factor maintaining the cardiac phosphocreatine level as an energy storage in the heart. A reduction in the phosphocreatine/adenosine triphosphate ratio was found to precede the decreasing contractility of cardiac muscle in diabetic patients (31). These data suggested that increased BNIP3 could cause mitochondrial dysfunction in abnormal cardiac muscle energy metabolism, reduce mitochondrial efficiency, increase oxidative stress and decrease myocardial energy storage. These changes precede cellular apoptosis and play an indispensable role in myocardial dysfunction.

The present results also showed that BNIP3 could negatively regulate the MMP9 expression level. MMP9 denatures fibronectin and fibrillar collagen (32). Accumulation of these extracellular proteins promotes intercellular fibrosis and cardiac stiffness; therefore, elevated BNIP3 expression in high-glucose conditions induced intercellular fibrosis by inhibiting MMP9, and hence decreased myocardial compliance.

The observation of increased mitochondrial fusion in cells overexpressing BNIP3 was unexpected. Mitochondrial fusion has been shown to be critical for maintaining normal mitochondrial function in response to metabolic or environmental stress (33). Increased mitochondrial fusion may be associated with an increased requirement for oxidative phosphorylation depending on lipid metabolism (33). Furthermore, mitochondrial fission may contribute to apoptosis induced by hyperglycemia (34). The mechanisms underlying the BNIP3 overexpression-enhanced mitochondrial fusion require further investigation.

DCM was first recognized in 1955 (35) and defined in 1972 (7) as a disease with heart dilation dysfunction with or without systolic dysfunction. Histologically, the ventricular dilation is associated with cardiomyocyte hypertrophy, as well as intercellular fibrosis. Chronic hyperglycemia in diabetes alters substrate metabolism to cause mitochondrial dysfunction, stimulate oxidative stress and calcium overload, and increase the glycation of interstitial proteins (8). Notably, it was suggested in the present study that a number of these pathways, including altered substrate metabolism, increased oxidative stress and regulation of interstitial proteins, are potentially regulated by BNIP3 in response to hyperglycemia. We therefore hypothesized that, in addition to promoting mitochondria-mediated cellular apoptosis, BNIP3 could represent an important intracellular signaling mechanism mediating the effect of hyperglycemia in cardiac muscle metabolism. BNIP3 may, therefore, provide a potential pharmaceutical target in DCM. Further studies are required to confirm the present observations.

\section{Acknowledgements}

This study was financially supported by the Science and TechnologyCommissionofShanghaiMunicipality,Experimental Animal Research Program (no. 11140900500.11140900502), the Pujiang Program (no. 12PJ1407700) and the National Natural Science Foundation of China (no. NFSC81200563).

\section{References}

1. Guariguata L: By the numbers: new estimates from the IDF Diabetes Atlas Update for 2012. Diabetes Res Clin Pract 98: 524-525, 2012.

2. Xu Y, Wang L, He J, et al; 2010 China Noncommunicable Disease Surveillance Group: Prevalence and control of diabetes in Chinese adults. JAMA 310: 948-959, 2013.

3. Fox CS, Coady S, Sorlie PD, et al: Trends in cardiovascular complications of diabetes. JAMA 292: 2495-2499, 2004.

4. No authors listed: Intensive blood-glucose control with sulphonylureas or insulin compared with conventional treatment and risk of complications in patients with type 2 diabetes (UKPDS 33). UK Prospective Diabetes Study (UKPDS) Group. Lancet 352: 837-853, 1998. 
5. Poirier P, Bogaty P, Garneau C, Marois L and Dumesnil JG: Diastolic dysfunction in normotensive men with well-controlled type 2 diabetes: importance of maneuvers in echocardiographic screening for preclinical diabetic cardiomyopathy. Diabetes Care 24: 5-10, 2001

6. Bertoni AG, Tsai A, Kasper EK and Brancati FL: Diabetes and idiopathic cardiomyopathy: a nationwide case-control study. Diabetes Care 26: 2791-2795, 2003.

7. Rubler S, Dlugash J, Yuceoglu YZ, Kumral T, Branwood AW and Grishman A: New type of cardiomyopathy associated with diabetic glomerulosclerosis. Am J Cardiol 30: 595-602, 1972.

8. Fonarow GC and Srikanthan P: Diabetic cardiomyopathy. Endocrinol Metab Clin North Am 35: 575-599, 2006.

9. Young ME, McNulty $\mathrm{P}$ and Taegtmeyer H: Adaptation and maladaptation of the heart in diabetes: Part II: potential mechanisms. Circulation 105: 1861-1870, 2002.

10. Boudina S and Abel ED: Diabetic cardiomyopathy revisited Circulation 115: 3213-3223, 2007.

11. Crow MT, Mani K, Nam YJ and Kitsis RN: The mitochondrial death pathway and cardiac myocyte apoptosis. Circ Res 95: 957-970, 2004.

12. Modrak J: Collagen metabolism in the myocardium from streptozotocin-diabetic rats. Diabetes 29: 547-550, 1980.

13. Shimizu M, Umeda K, Sugihara N, et al: Collagen remodelling in myocardia of patients with diabetes. J Clin Pathol 46: 32-36, 1993.

14. Neubauer S: The failing heart - an engine out of fuel. N Engl J Med 356: 1140-1151, 2007.

15. Crow MT: Hypoxia, BNip3 proteins, and the mitochondrial death pathway in cardiomyocytes. Circ Res 91: 183-185, 2002.

16. Yasuda M, Theodorakis P, Subramanian T and Chinnadurai G Adenovirus E1B-19K/BCL-2 interacting protein BNIP3 contains a BH3 domain and a mitochondrial targeting sequence. J Biol Chem 273: 12415-12421, 1998.

17. Lomonosova $\mathrm{E}$ and Chinnadurai G: $\mathrm{BH} 3$-only proteins in apoptosis and beyond: an overview. Oncogene 27 Suppl 1: S2-S19, 2008.

18. SulistijoES, JaszewskiTM and MacKenzie KR: Sequence-specific dimerization of the transmembrane domain of the 'BH3-only' protein BNIP3 in membranes and detergent. J Biol Chem 278: 51950-51956, 2003.

19. Bocharov EV, Pustovalova YE, Pavlov KV, et al: Unique dimeric structure of BNip3 transmembrane domain suggests membrane permeabilization as a cell death trigger. J Biol Chem 282: 16256-16266, 2007.

20. Ray R, Chen G, Vande Velde C, et al: BNIP3 heterodimerizes with $\mathrm{Bcl}-2 / \mathrm{Bcl}-\mathrm{X}(\mathrm{L})$ and induces cell death independent of a Bcl-2 homology 3 (BH3) domain at both mitochondrial and nonmitochondrial sites. J Biol Chem 275: 1439-1448, 2000.
21. Guo K, Searfoss G, Krolikowski D, et al: Hypoxia induces the expression of the pro-apoptotic gene BNIP3. Cell Death Differ 8: 367-376, 2001.

22. Regula KM, Ens K and Kirshenbaum LA: Inducible expression of BNIP3 provokes mitochondrial defects and hypoxia-mediated cell death of ventricular myocytes. Circ Res 91: 226-231, 2002.

23. Diwan A, Krenz M, Syed FM, et al: Inhibition of ischemic cardiomyocyte apoptosis through targeted ablation of Bnip3 restrains postinfarction remodeling in mice. J Clin Invest 117: 2825-2833, 2007.

24. Zhang F, Li G, Ding W, et al: Screening and analysis of early cardiopathology-related gene in type 2 diabetes mellitus. Zhonghua Nei Ke Za Zhi 41: 530-533, 2002 (In Chinese).

25. Zhang F, Ye C, Li G, et al: The rat model of type 2 diabetic mellitus and its glycometabolism characters. Exp Anim 52: 401-407, 2003.

26. Althaus J,Bernaudin M,PetitE, Toutain J, Touzani O and Rami A: Expression of the gene encoding the pro-apoptotic BNIP3 protein and stimulation of hypoxia-inducible factor-1alpha (HIF-1alpha) protein following focal cerebral ischemia in rats. Neurochem Int 48: 687-695, 2006.

27. Papandreou I, Krishna C, Kaper F, Cai D, Giaccia AJ and Denko NC: Anoxia is necessary for tumor cell toxicity caused by a low-oxygen environment. Cancer Res 65: 3171-3178, 2005.

28. Kim JY, Cho JJ, Ha J and Park JH: The carboxy terminal C-tail of BNip3 is crucial in induction of mitochondrial permeability transition in isolated mitochondria. Arch Biochem Biophys 398: 147-152, 2002.

29. Vande Velde C, Cizeau J, Dubik D, et al: BNIP3 and genetic control of necrosis-like cell death through the mitochondrial permeability transition pore. Mol Cell Biol 20: 5454-5468, 2000.

30. Minhas KM, Saraiva RM, Schuleri KH, et al: Xanthine oxidoreductase inhibition causes reverse remodeling in rats with dilated cardiomyopathy. Circ Res 98: 271-279, 2006.

31. Duncan JG: Mitochondrial dysfunction in diabetic cardiomyopathy. Biochim Biophys Acta 1813: 1351-1359, 2011.

32. Spinale FG: Myocardial matrix remodeling and the matrix metalloproteinases: influence on cardiac form and function. Physiol Rev 87: 1285-1342, 2007.

33. Youle RJ and van der Bliek AM: Mitochondrial fission, fusion, and stress. Science 337: 1062-1065, 2012.

34. Yu T, Sheu SS, Robotham JL and Yoon Y: Mitochondrial fission mediates high glucose-induced cell death through elevated production of reactive oxygen species. Cardiovasc Res 79: 341-351, 2008.

35. Ungar I, Gilbert M, Siegel A, Blain JM and Bing RJ: Studies on myocardial metabolism. IV. Myocardial metabolism in diabetes. Am J Med 18: 385-396, 1955. 